\title{
Knowing Who's Boss: Implicit Perceptions of Status From the Nonverbal Expression of Pride
}

\author{
Azim F. Shariff and Jessica L. Tracy \\ University of British Columbia
}

\begin{abstract}
Evolutionary theory suggests that the universal recognition of nonverbal expressions of emotions functions to enhance fitness. Specifically, emotion expressions may send survival-relevant messages to other social group members, who have the capacity to automatically interpret these signals. In the present research, we used 3 different implicit association methodologies to test whether the nonverbal expression of pride sends a functional, automatically perceived signal about a social group member's increased social status. Results suggest that the pride expression strongly signals high status, and this association cannot be accounted for by positive valence or artifacts of the expression such as expanded size due to outstretched arms. These findings suggest that the pride expression may function to uniquely communicate the high status of those who show it. Discussion focuses on the implications of these findings for social functions of emotion expressions and the automatic communication of status.
\end{abstract}

Keywords: social status, emotion expressions, implicit signals, pride

Although the bulk of research on emotion expressions has examined how these expressions are recognized and distinguished from one another (e.g., Keltner \& Ekman, 2000), an equally longstanding but less extensively researched line of inquiry has examined the ultimate function of each expression (see, e.g., Cosmides \& Tooby, 2000; Darwin, 1872; Ekman, 1992). In this vein, most expressions have been assumed to serve a communicative function; fear, for example, transmits a rapidly and universally interpreted signal informing onlookers of important environmental events (Ohman, 2002; Ohman \& Mineka, 2001). Indeed, the large body of cross-cultural recognition data on emotion expressions (e.g., Ekman \& Friesen, 1971; Ekman et al., 1987) is consistent with the claim that these expressions serve communicative functions. However, relatively little empirical work has addressed the question of what, precisely, each expression communicates, beyond knowledge of the emotion displayed. From an evolutionary perspective, humans should be expected not only to show accurate and quick recognition of the particular emotion

Azim F. Shariff and Jessica L. Tracy, Department of Psychology, University of British Columbia.

We are grateful to the Social Sciences Research Council of Canada for supporting this research (File 767-2006-1980 and 410-2006-1593), and a Michael Smith Foundation for Health Research Scholar award, CI-SCH01862 (07-01). We thank Lara Aknin, Vanja Alispahic, Emma Buchtel, Joey Cheng, Lesley Duncan, and Christine Prehn for their helpful comments on the paper, and Jeff Markusoff for his help with the study design, data collection, and comments on the paper. We especially thank the UBC Psychobiological Determinants of Health Laboratory and Eva Zysk in particular for developing and validating the abstract painting stimuli used in Study 4.

Correspondence concerning this article should be addressed to Azim F. Shariff, Department of Psychology, University of British Columbia, 2136 West Mall, Vancouver, B.C., Canada, V6T 1Z4. E-mail: azim@psych.ubc.ca expressed (e.g., "fear"), but they also should be able to decode what each expression means (e.g., "Danger!").

Those studies that have examined the meaningful content conveyed by emotion expressions have largely focused on the broad distinction between positive and negative expressions, demonstrating that the former typically send approach-oriented messages, and the latter more avoidance-oriented messages (e.g., Mogg \& Bradley, 1999). Further research has shown that these approach/ avoidance messages can be perceived even when expressions are displayed subliminally, suggesting a nonconscious perception process (Winkielman \& Berridge, 2003).

\section{Perceptions of Status and the Pride Expression}

Several researchers have suggested that the "self-conscious" emotion of pride evolved, in part, to convey information about an individual's social status (Fessler, 1999; Tracy \& Robins, 2007a; Tracy, Shariff, \& Cheng, in press). Given the importance among group-living primates of effectively navigating one's social environment, the ability to communicate and identify status hierarchies is likely to be highly adaptive (Sedikides \& Skowronski, 1997). In particular, individuals who can effectively communicate that they deserve increased social status may be more likely to receive the benefits associated with high status, including access to scarce resources, increased influence, and higher quality mates. In addition, the ability to ascertain who in one's social group deserve high status would facilitate power maneuvering, cooperation, and generally smoother social interactions (e.g., displaying appropriate deference, making worthwhile alliances; Eibl-Eibesfeldt, 1989; Schmid Mast \& Hall, 2004).

Although no studies have directly examined whether the nonverbal pride expression is associated with high status, several lines of research are consistent with this expectation. First, high-status individuals have been found to report greater momentary feelings of pride than low-status individuals, after succeeding at the same task, suggesting an experiential association between high status 
and pride (Tiedens, Ellsworth, \& Mesquita, 2000; Tiedens, Ellsworth \& Moskowitz, 2000). Second, pride feelings have been shown to motivate behaviors that make high status particularly likely; individuals induced to feel pride exhibit greater perseverance on difficult tasks, which in turn may promote achievement and consequent high status (Williams \& DeSteno, 2008, 2009); in fact, a recent study found that individuals manipulated to experience pride were subsequently perceived as behaving in a dominant manner in a group task (Williams \& DeSteno, 2009). Third, individuals across cultures have been shown to respond to socially valued achievements-events that likely increase one's status-by displaying a distinct nonverbal expression that is reliably and cross-culturally associated with pride (Lewis, Alessandri, \& Sullivan, 1992; Tracy \& Matsumoto, 2008; Tracy \& Robins, 2004, 2008b; Weisfeld \& Beresford, 1982). This behavioral response to success, which includes an expanded posture, head-tilt back, and arms extended out from the body, seems to be biologically innate, based on evidence from congenitally blind individuals (Tracy \& Matsumoto, 2008). Moreover, the morphological similarity of the pride expression to the dominance displays of nonhuman primates (e.g., de Waal, 1989; Tracy \& Robins, 2007b), suggests that it might have evolved from earlier displays that served a similar function in establishing status hierarchies. Despite this growing body of circumstantial evidence, however, no previous research has directly examined whether the nonverbal expression of pride promotes perceptions of high status in those who show it.

\section{The Present Research}

Seven studies using (a) the implicit association test (IAT; Greenwald \& Banaji, 1995), (b) the single-target IAT (Penke, Eichstaedt, \& Asendorpf, 2006), and (c) the affect misattribution procedure (AMP; Payne, Cheng, Govorun, \& Stewart, 2005), tested whether the pride expression functions as an automatic signal of high status. The standard IAT, used in Studies 1, 4, 5, and 6 , assesses implicit associations between pairs of dichotomous stimuli by measuring reaction times for categorizing stimuli across pairings. The results of these IATs are necessarily relative; that is, if pride is associated with high status, the IAT will reveal this by demonstrating that the association between pride and high status is stronger than associations between some other target and high status. These standard IATs thus were supplemented with the nonrelative single-target IAT and the AMP, both of which can demonstrate an implicit association between pride and high status irrespective of other associations. Responses to all three of these tasks can be considered automatic associations insofar as they meet the criteria for two of the "four horsemen" of automaticity: lack of intention and lack of control (cf. Bargh, 1994). Given that participants in these tasks are specifically instructed to respond in a way that would yield null differences between conditions (see procedure descriptions below), any significant differences that emerge can be assumed to have occurred without intention or control. Furthermore, response differences that emerge in the AMP can be further claimed to lack some level of awareness because AMP study participants are unaware of the influence of the target stimuli (in this case, the pride expression; see procedure description below) on subsequent judgments - a necessary precondition for any misattribution (Payne et al., 2005).
In addition to providing the first test of whether the pride expression is an automatic signal of status, this research extends previous findings in several ways. First, these studies test whether distinct positive emotion expressions serve distinct functions. If the pride expression is associated with a status-relevant signal that is not conveyed by happiness, it would support claims that pride and happiness have distinct expressions that serve divergent functions (e.g., Tracy \& Robins, 2004). Such a finding would provide a more nuanced understanding of the previously documented broad association between power and positive affect (Keltner, Anderson, \& Gruenfeld, 2003), and would raise challenges for the argument that most of the distinctions made among various positive emotions, and among various negative emotions, are purely cognitive conceptualizations (Barrett, 2006; Russell, 2003).

Second, this is the first research to test whether emotion expressions underlie the automatic communication of status. Given the predominance of status hierarchies in everyday life, and the frequency and ease with which individuals perceive and respond to status differences (Schmid Mast \& Hall, 2004), it is likely that humans infer others' status rapidly and automatically. However, it is unclear how this is performed. The pride expression may provide one answer, having evolved as part of an adaptive system for signaling and detecting status increases. If this is the case, findings from the present research would highlight the nonverbal behavioral and emotional mechanism that underlies the conferral of status in many everyday social interactions.

A third contribution of this research is its novel application of the IAT and the AMP to examine the signaling function of an emotion expression. Both methods have previously been used to examine a wide range of attitudes and preferences, including implicit prejudice (Greenwald, McGhee, \& Schwartz, 1998), selfesteem (Greenwald \& Farnham, 2000), consumer preferences (Maison, Greenwald, \& Bruin, 2004), and political voting behavior (Payne et al., 2005); but neither has been used to test a functionalist account of emotion expressions. Given the lack of previous studies on the functions of distinct emotion expressions, this application may prove a promising innovation for future research.

\section{Study 1}

Study 1 examined whether the nonverbal expression of pride is more strongly associated with high status than are the nonverbal expressions of two other self-conscious emotions-shame and embarrassment. Shame and embarrassment expressions may function to signal low status (e.g., Fessler, 1999; Keltner, 1995; Keltner \& Buswell, 1997) so, theoretically, the pride expression should be more strongly associated with high status than these other expressions. If no difference is found between the expressions of pride and embarrassment/shame in their respective associations with high status, it would challenge our account of pride as having evolved to uniquely signal high status.

Participants completed an IAT that paired photographs of pride expressions or photos of shame and embarrassment expressions with words representing high or low status, and we compared the speed of their responses to presumed congruent versus incongruent pairings. If participants are faster to respond to the presumed congruent pairing (pride with high status and shame/embarrassment with low status) than to the incongruent pairing (pride with low status and shame/embarrassment with high status), it would 
indicate a stronger implicit association between the pride expression and high status than pride and low status, or between shame/ embarrassment expressions and low status than shame/embarrassment and high status, or both. One benefit of this approach is that participants are told to categorize all stimuli equally quickly (as quickly as possible), so, if pride is associated with high status, individuals' responses will demonstrate this fact despite their conscious efforts not to do so, indicating that the pride-signaling process occurs without intention and cannot be controlled.

\section{Method}

\section{Participants and Procedure}

Twenty undergraduate students (80\% women) participated in exchange for course credit. Participants were seated at a computer with a 17" monitor, and viewed three photos of an actor displaying the pride expression, then three photos of the same actor displaying the shame or embarrassment expression. The three pride expressions were explicitly labeled "Position A", and the three shame/ embarrassment expressions were explicitly labeled "Position B". Shame and embarrassment expressions were included within a single set because these two expressions may not be distinct cross-culturally (Edelstein \& Shaver, 2007; Haidt \& Keltner, 1999), and they likely serve a similar signaling function that is opposite to that of pride. Participants were given no indication that the positions conveyed emotion expressions.

Participants next completed an IAT pairing these photos with words representing either high or low status. They categorized the photos into the appropriate position (A or B), and the words as either high- or low-status, by pressing one of two keys (accuracy rate for single-categorization tasks $=93 \%$ for photos, $95 \%$ for words). In one critical testing block, pride photos and high-status words shared a key, and shame/embarrassment photos and lowstatus words shared a key. In the critical comparison block, these pairings were reversed (i.e., pride and low-status shared a key, and shame/embarrassment and high-status shared a key). The order of these pairings was counterbalanced between participants.

\section{Stimuli}

Expressions. Photographs depicted a White male showing three variations of pride, one variation of shame (the only expression known to reliably convey shame), and two variations of embarrassment. ${ }^{1}$ All variations have been demonstrated to reliably convey pride, shame, and embarrassment, respectively ${ }^{2}$ (Haidt \& Keltner, 1999; Keltner, 1995; Tracy \& Robins, 2007b).

Words. The high-status words presented were commanding, dominant, important, powerful, and prestigious, and the low-status words presented were humble, minor, submissive, unimportant, and weak. We derived these words by obtaining 27 synonyms or antonyms for "high status" and "low status" from an online thesaurus (Kipfer, 2005), and asking nine upper level undergraduate psychology students to rate each word for its relevance to high and low status, separately, using 5-point Likert scales ranging from 1 (I definitely would not call this high/low status) to 5 (I definitely would call this high/low status; $\alpha \mathrm{s}=.96, .93$, for high and low-status ratings, respectively). The five highest and lowest status words, all of which had mean ratings of 4.5 or greater on the highor low-status scales, respectively, were retained.

\section{Results and Discussion}

We calculated implicit associations in accordance with the scoring algorithm proposed by Greenwald, Nosek, and Banaji (2003). Specifically, we first excluded responses that exceeded $10 \mathrm{~s}$, given that participants who responded this slowly were likely not attending to the task. Second, because error rates vary systematically with implicit associations, we added a $15 \mathrm{~ms}$ time penalty to the mean trial time for each incorrect response (rather than simply excluding trials in which expressions or words were incorrectly categorized). ${ }^{3}$ We then calculated difference scores for each individual from these error-adjusted means, by dividing the difference between their mean reaction times for the two pairings of interest by their overall standard deviations for the two pairings. As a result, each participant was given a difference score representing the strength and direction of his or her implicit association. We conducted one-sample $t$ tests to determine whether these $d$ scores differed significantly from zero-which would suggest that differences in reaction times between pairings were meaningful. For all studies, we report mean $d$ scores (Greenwald, Nosek, \& Banaji, 2003) across participants, one-sample $t$ test statistics, and more traditional Cohen's $d$ s that indicate the overall sample effect sizes.

In Study 1, participants showed a strong implicit association, with substantially faster reaction times when pride expressions were paired with high-status words and shame/embarrassment expressions were paired with low-status words than when pride expressions were paired with low-status words and shame/ embarrassment expressions were paired with high-status words, d score $=0.66, t(19)=5.56, p<.05$, Cohen's $d=2.55$ (see Figure 1 ). Given that this difference emerged despite participants' conscious efforts to respond equally quickly on every trial, it suggests that, at an implicit level, the pride expression is more strongly associated with high status than the shame/embarrassment expressions, or that the shame/embarrassment expressions are more strongly associated with low status than the pride expression, or that both associations exist.

To determine whether an association between pride and high status was responsible for the effect that emerged in Study 1, we conducted Study 2, in which we paired the same high- and low-

\footnotetext{
${ }^{1}$ The pride expressions differed in arm position; one included arms raised above the head with hands in fists, one included arms akimbo with hands on hips, and one included arms crossed on the chest; all three are reliably recognized as pride (Tracy \& Robins, 2007b). The embarrassment expressions were identical except that one showed the actor covering part of his face with his hand; both variants are reliably recognized as embarrassment (Haidt \& Keltner, 1999; Heerey, Keltner, \& Capps, 2001).

${ }^{2}$ Emotion expressions shown in all studies, with the exception of the comparison expressions shown in Study 6, were verified with the EmotionFacial Action Coding System (EMFACS; Ekman \& Rosenberg, 1998), by a certified FACS expert (Ekman \& Friesen, 1978). These photos were taken from the University of California Davis Set of Emotion Expressions (UCDSEE; Tracy, Robins, \& Schriber, 2009); all included the same actor photographed from the waist up. All photos used in all studies can be obtained by request from the first author.

${ }^{3}$ For example, if a participant averaged $700 \mathrm{~ms}$ on a particular block, and made three mistakes, the error-corrected average trial time would be $700 \mathrm{~ms}+(3 \times 15 \mathrm{~ms})=745 \mathrm{~ms}$. This is equivalent to adding $600 \mathrm{~ms}$ per error to the sum total of the 40 trials prior to averaging, as was recommended by Greenwald et al. (2003).
} 


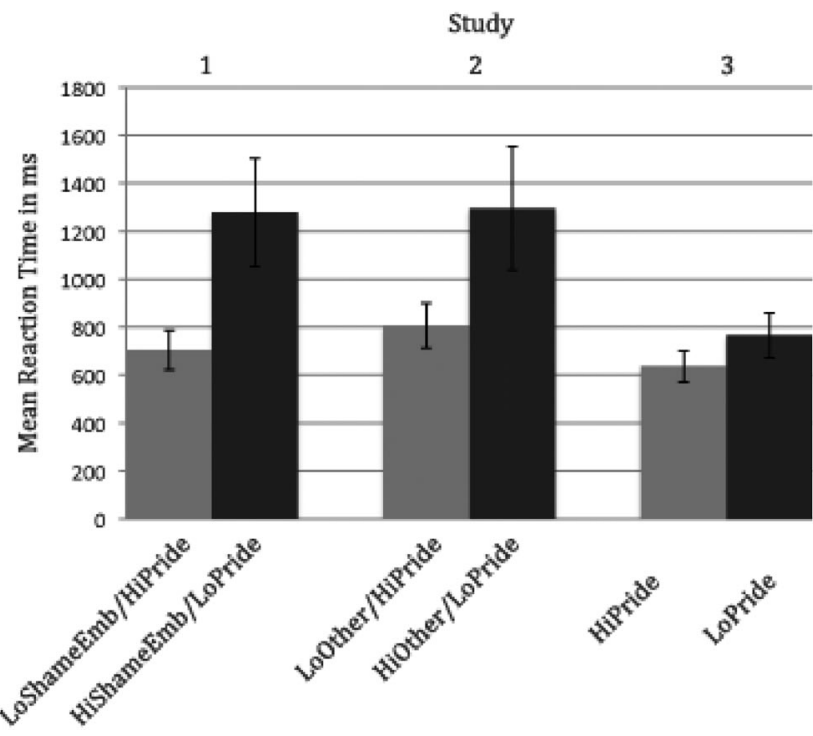

Figure 1. Overall error-adjusted mean reaction times across participants, separately for Studies 1, 2, and 3. Higher (i.e., slower) reaction times indicate a weaker association. These means should be treated as a crude measure of implicit association test effects because, unlike the d scores reported in text, they do not account for individual variability, although they do account for errors. Error bars indicate standard error of the mean. HiPride/LoShameEmb $=$ the condition in which pride expressions were paired with high-status words, and shame/embarrassment expressions were paired with low-status words; this condition was compared with the HiShameEmb/LoPride condition in which shame/embarrassment expressions were paired with high-status words and pride expressions with low-status words; other $=$ the combination of disgust, fear, and happy expressions. The corresponding $d$ scores of all three comparisons are significant.

status words with either pride expressions or a set of three photos of "other" emotions, not representing pride, shame, or embarrassment. If reaction times are still faster when pride is paired with high-status words, we can conclude that the results of Study 1 were at least partly due to an association between pride and high status, given that the "other" emotions are not theoretically associated with low status.

\section{Study 2}

\section{Method}

Forty undergraduates (20 in each study; $80 \%$ women) participated in exchange for course credit. The method was the same as in Study 1, except that pride expressions were paired against three photos of the same actor displaying disgust, fear, and happiness expressions (accuracy rate for photo categorization $=96 \%$ ). These three expressions were chosen because they represent a mixture of positive and negative emotions that likely send messages distinct from each other and from pride, and none of the three is theoretically associated with high or low status.

\section{Results and Discussion}

A strong implicit association emerged between the pride expression and high status. Mean reaction times were faster when pride expressions were paired with high-status words and "other" expressions were paired with low-status words than the reverse pairings, d score $=0.60, t(19)=4.72, p<.05, d=2.36$ (see Figure 1). This finding indicates that the implicit association that emerged in Study 1 was at least partly driven by an association between the pride expression and high status and not entirely by an association between shame/embarrassment expressions and low status. However, given the necessarily relative nature of the standard IAT, we cannot be certain that the effect found here is due to pride, per se. It remains possible that the other emotion set was implicitly associated with low status, and this association accounted for the differences that emerged. Although we included three fairly different expressions in the other set, the inclusion of fear-an expression that may signal submission - could have been salient enough to drown out any opposing impact of happiness and disgust, and promote a low-status association with the set as a whole. Thus, in Study 3, we used the nonrelative, single-target IAT (Penke et al., 2006) to assess whether pride is associated with high status irrespective of any comparison stimulus.

\section{Study 3 \\ Method}

Twenty undergraduate students ( $80 \%$ women) participated in exchange for course credit. Procedures were the same, except that instead of the standard relative IAT, participants completed a single-target IAT. As with standard IAT, the single-target IAT used the dichotomous attribute categories of high and low status, but included only one target variable-photos of the pride expression. Scores were obtained by comparing reaction times between trials in which pride was paired with high-status words and trials in which pride was paired with low-status words.

\section{Results and Discussion}

The scoring of the single-target IAT was virtually identical to the scoring of the standard IAT. Error corrected mean reaction times for pride-high-status pairings were subtracted from errorcorrected means for pride-low-status pairings, and the difference was divided by the standard deviation of the reaction times of both trials. Positive $d$ scores, therefore, indicate relatively higher (slower) reaction times for pride-low-status pairings compared to pride-high-status pairings, and thus a stronger (faster) relationship between pride and high status.

A strong positive mean $d$ score of 0.22 emerged $t(19)=4.41$, $d=2.02, p<.05$. Given that the pride expression was not compared to any other expression in this study, these results suggest that the pride expression implicitly evokes the concept of high status irrespective of any other emotion. Furthermore, given that even low-status words are associated with the broader concept of status, we might have expected no difference, or at least a weaker effect, in this study. The fact that the effect size remained large highlights the strength of the specific association between pride and high status.

Although this study addresses one of the most prominent shortcomings of the standard IAT - its necessarily relative natureother limitations remain. Most of these concerns (e.g., participants' ability to fake responses, the impact of individual differences in 
executive control; Payne, 2005; Tierney, 2008; but see also Blanton et al., 2009) are more relevant to studies assessing undesirable biases (e.g., racism) or individual differences in implicit associations, than they are to the present research goals. Nonetheless, we conducted an additional study using a non-IAT method for assessing implicit responding, to more firmly establish the implicit association between pride and high status. Specifically, in Study 4, we sought to replicate the results of Studies 1, 2, and 3 using a measure of implicit associations that has been shown to ameliorate these concerns: the affect misattribution procedure (AMP; Payne et al., 2005).

\section{Study 4}

\section{Method}

Thirty-five undergraduate students ( $80 \%$ women) participated in exchange for course credit. As in Studies 1, 2, and 3, participants were seated at a 17 " computer monitor, and all task instructions appeared onscreen. The AMP was developed to measure implicit preferences by creating a situation in which participants might reveal such preferences (e.g., attitudes toward African Americans) by misattributing them to an otherwise neutral stimulus (e.g., Chinese ideographs; Payne et al., 2005). In the present study, instead of focusing on affective liking or disliking responses, we used the AMP to measure a more complex misattribution- - high status. Using the standard AMP procedure, we tested whether participants would misattribute their status associations with the pride expression to a neutral stimulus. To our knowledge, no previous published study has used the AMP to assess associations other than preferences.

Participants were briefly but supraliminally $(75 \mathrm{~ms})$ primed with a photo of the actor showing the pride expression, or with a photo of a neutral control stimulus (a gray box) and then, again briefly but supraliminally (100 ms), shown an image of an abstract painting (see Figure 2). Each image was followed by a visual mask, consistent with Payne et al. (2005; see Figure 2). After viewing the abstract painting image, participants were asked to press one key if they felt the painting was "lower status than average," and another key if they felt it was "higher status than average". Following Payne et al., participants were given explicit instruc-

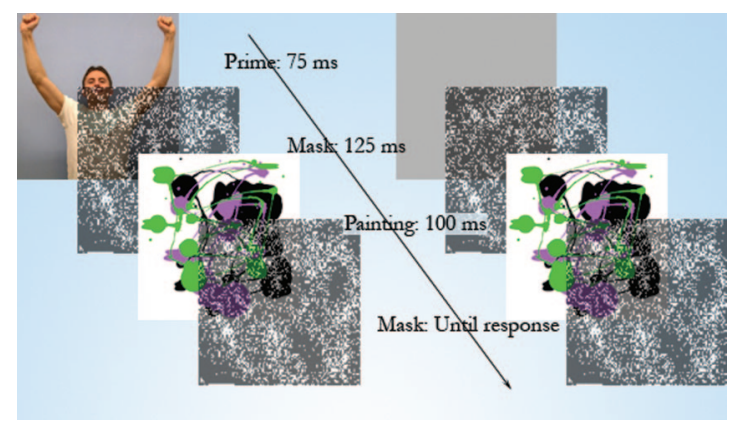

Figure 2. Sequential order of the priming procedure used in Study 4. Participants viewed the brief but supraliminal image of either the pride expression (top left) or a neutral gray box (top right). The abstract painting, which participants were asked to classify as high or low status, was then displayed between two masks. tions before the task began to ignore the priming photos (pride expressions or neutral images), and to base their status impression solely on the abstract art. Of note, typical AMP studies use Chinese ideographs as the neutral stimulus on to which participants are led to misattribute their preferences (e.g., Payne et al., 2005), but we were concerned that a number of our participants (students at the University of British Columbia, which has a high Asian population) would be familiar with the meaning of these ideographs and thus have prior, nonneutral, associations that would interfere with the AMP's intended purpose. Thus, we instead used computer-generated abstract paintings, which were similar to the work of Jackson Pollack. ${ }^{4}$ If the pride expression is implicitly associated with high status, the logic behind the AMP predicts that this association be misattributed to the paintings, such that paintings following the pride expression would more frequently be perceived as high status than paintings following the control (gray box) prime.

\section{Results and Discussion}

To calculate a pride-high-status association score, we subtracted the proportion of times paintings following the control stimulus were perceived as high status from the proportion of times paintings following the pride expression were perceived as high status; this yielded a score between 1 and -1 for each participant, where 0 represents chance-meaning that participants were equally likely to rate paintings following either target stimulus as high status. Positive scores indicate a greater association between pride and high status, with a maximum score of +1 indicating that every painting following the pride expression was rated as high status, and every painting following the control stimulus was rated as low status.

The mean score across all participants was +.17 , which was significantly greater than chance based on the one-sample $t$ test, $t(34)=3.98, d=1.37, p<.05$. Furthermore, as can be seen from Figure 3, the large majority of participants (76\%) showed a positive score, indicating an association between the pride expression and high status. Thus, using the AMP, we again found a strong implicit association between the pride expression and high status, and, together, these four studies converge to provide robust support for an implicit association between the pride expression and high status.

However, given that pride is a positively valenced emotion, it remains possible that shared associations between high status and positive valence account for the association. Although happiness was included in the other set of expressions contrasted with pride in Study 2, its positivity may have been overwhelmed by the negativity of the two other expressions in that set. Thus, it remains possible that the high-status signal sent by pride is not unique to pride, but rather is shared by other positive emotion expressions such as happiness. It also remains possible that pride is one of several high-power, status-related emotions, along with angeranother emotion known to convey a sense of power or competence (Tiedens, 2001). If this is the case, the high-status implicit associations of the pride expression would not be due to its unique signaling function, but rather to a broader dimensional property of

\footnotetext{
${ }^{4}$ Available from http://www.jacksonpollock.org/
} 
pride (Knutson, 1996). Thus, we conducted Studies $5 \mathrm{a}$ and $5 \mathrm{~b}$ to examine whether the pride expression is a stronger signal of high status than the happiness (Study 5a) and anger (Study 5b) expressions.

\section{Studies $5 \mathrm{a}$ and $5 \mathrm{~b}$}

\section{Method}

Forty undergraduates ( $80 \%$ women, 20 per study) participated in exchange for course credit. We used the standard IAT methodology, as in Studies 1 and 2, but pride photos were compared with three photos displaying the happiness expression (Study 5a; accuracy rate for pride/happy photo categorization $=96 \%),{ }^{5}$ or with three photos displaying the anger expression (Study 5b; accuracy rate for the pride/anger photo categorization $=96 \%) .{ }^{6}$ All expressions were posed by the same actor.

\section{Results and Discussion}

In both studies, strong implicit associations emerged between the pride expression and high status. In Study 5a, reaction times were significantly faster when pride expressions were paired with high-status words and happiness expressions were paired with low-status words than the reverse pairings, $d$ score $=0.65$, $t(19)=5.44, d=2.50, p<.05$ (see Figure 4). This finding suggests that pride is more strongly associated with high status than is happiness, and that the association between pride and high status is not solely due to positive valence. If the association was due to shared positivity, then happiness should have been equally (if not more) strongly associated with high status, compared with pride.

In Study $5 b$, reaction times were significantly faster when pride expressions were paired with high-status words, and anger expressions were paired with low-status words, than the reverse pairings, $d$ score $=0.45, t(19)=4.42, d=1.93, p<.05$. It is unlikely that this effect was driven by an association between

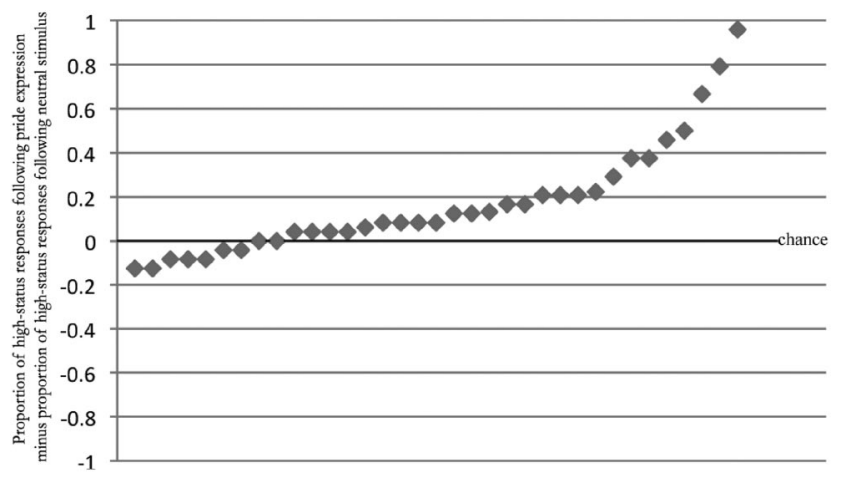

Figure 3. Distribution of scores on the affective misattribution procedure. Each data point represents one participant. Positive scores indicate a greater proportional likelihood of classifying a painting as higher status than average following the brief presentation of the pride expression, compared to the brief presentation of a neutral stimulus. Twenty-six of 34 participants $(76 \%)$ scored above zero, indicating a greater implicit association between the pride expression and high status than between the neutral stimulus and high status.

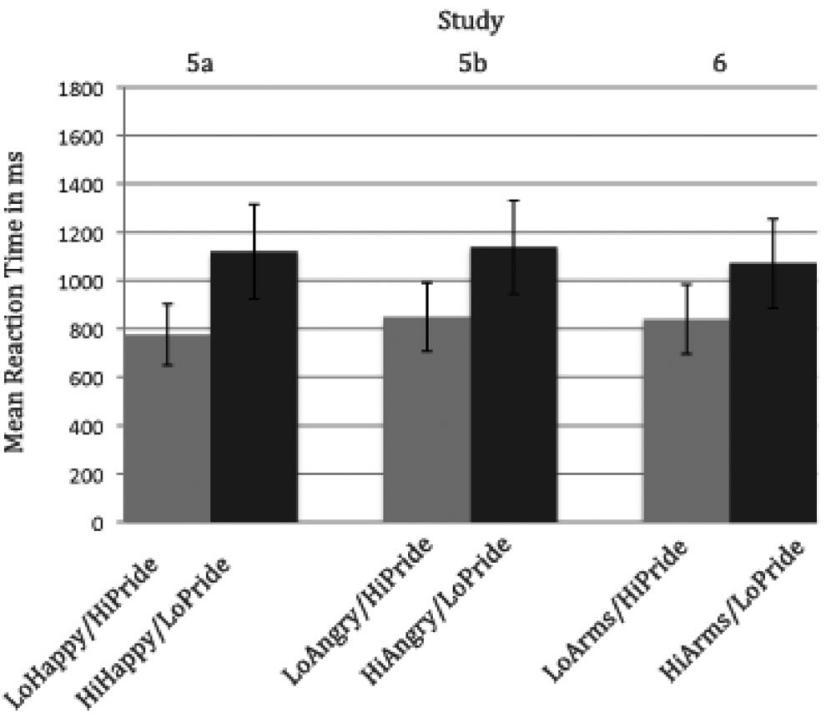

Figure 4. Overall error-adjusted mean reaction times across participants, separately for Studies 5a, 5b, and 6. Higher (i.e., slower) reaction times indicate a weaker association. Error bars indicate standard error of the mean. Lo = low-status words; Happy = happiness expressions; $\mathrm{Hi}=$ high-status words; Angry = anger expressions; Arms = neutral expressions with extended arms; HiPride/LoArms = the condition in which pride expressions were paired with high-status words, and photos of the target with extended arms were paired with low-status words, this condition was compared against the HiArms/LoPride condition in which the pairings were reversed. The corresponding $d$ scores of all three comparisons are significant.

anger and low status, given previous findings that anger expressions convey competence and that anger is a high-status emotion (Knutson, 1996; Tiedens, 2001). The findings from Studies $5 \mathrm{a}$ and $5 \mathrm{~b}$ thus suggest that pride is likely to be a unique signal of high status, or at least a stronger signal of high status than any other measured emotion-even those known to convey positivity and power.

However, there remains an additional alternative explanation for the pride-high-status association. In two of the three pride photos included, the actor's arms were extended out from his body, making him appear larger. In fact, increased largeness may be part of why the pride expression includes extended arms; size may be a heuristic for dominance or high status (Tracy \& Robins, 2007b). However, one could appear larger yet not show the pride expression (e.g., by waving one's arms); if the pride expression evolved as a gestalt signal of status, then it should convey status above and beyond other displays that also make individuals appear larger. To determine whether the effects found are artifacts of the expanded size in the pride expression, we conducted an additional study in which we compared pride expressions with photos of the target extending his arms out from his body, but showing no other components

\footnotetext{
${ }^{5}$ Two of these expressions showed the "Duchenne" smile, in which lip corners and cheek muscles are raised; they differed in intensity. The third photo showed a non-Duchenne smile with lip corners only raised.

${ }^{6}$ Two of the anger photos varied slightly in the precise configuration of action units; the third was a close-up version of one of these.
} 
of the pride expression. If the pride-high-status association is due to increased largeness or to the use of a heuristic involving extended arms, then including this comparison should remove the implicit association between pride and high status.

\section{Study 6}

\section{Method}

Twenty undergraduates ( $80 \%$ women) participated for course credit. Using the standard IAT methodology, photos of the pride expression were paired against three photos of the actor displaying a neutral facial expression while extending his arms in three different positions (accuracy rate for pride/arms photo categorization $=95 \%)^{7}$

\section{Results and Discussion}

A strong implicit association emerged between the pride expression and high status. Reaction times were significantly faster when pride expressions were paired with high-status words and extended-arms photos were paired with low-status words than the reverse pairings, $d$ score $=0.45, t(19)=4.40, d=2.0, p<.05$ (see Figure 4). This finding suggests that the previously documented implicit association between the pride expression and high status cannot be attributed solely to the extended arms or expanded size conveyed as part of the pride expression.

\section{General Discussion}

Together, these six studies provide strong support for a robust implicit association between the pride expression and the concept of high status. The association between pride and high status emerged when pride was compared with low-status emotionsshame and embarrassment-and when it was compared with a set of other emotions not theoretically relevant to status (disgust, fear, and happiness). This association also emerged when pride was directly compared with happiness and anger expressions, suggesting that the association cannot be accounted for by positive valence or a more general association between a range of emotions, including pride, and the dimension of power or status. Our final study comparing the pride expression with expressions in which the actor extended his arms out to appear larger demonstrates that the association between pride and high status is not the result of an artifact of the expression's appearance, such as expanded body size or outstretched arms.

Moreover, the finding of a strong association between pride and high status emerged from studies using three different methodologies, including one (the AMP) that assesses implicit associations without relying on reaction times. In all studies, the association between pride and high status was large-all Cohen's $d$ s exceeded 1.0 and most were greater than 2.0 - suggesting that the pride expression is a strong signal of high status.

\section{Implications}

The first major implication of this research is that the pride expression is a fairly specific signal of high status. Given the findings from Study 6, the pride display may be best viewed as a gestalt signal in which the sum of various parts (expanded body posture, small smile, head tilt back, arms extended) transmits an automatically interpreted message. However, it is also possible that the pride expression is more strongly associated with high status compared with the extended arms displays, or the happiness display, because it includes a greater number of pride-prototypical components. Future studies might address this issue by examining which components of the pride expression are necessary and sufficient to signal high status.

A second implication is that the pride expression sends a message that is distinct from that of happiness, consistent with a growing body of research suggesting that distinct positive emotions, such as pride and happiness, may have evolved to serve distinct functions (e.g., Shiota, Campos, Keltner, \& Hertenstein, 2004; L. A. Williams \& DeSteno, 2008). This finding challenges the view that distinctions within the category of positive emotions are cognitive conceptualizations not likely to be manifested in behavior (Barrett, 2006; Russell, 2003).

Third, the high-status signal sent by pride expression also appears to be distinct from any message sent by anger. Previous research has shown that displays of anger lead observers to judge individuals as more professionally qualified than individuals displaying sadness (Tiedens, 2001). Thus, anger seems to convey a sense of power or competence, at least relative to a low-power (and presumably low-status) emotion such as sadness. Yet, the present findings suggest that the anger expression does not convey high status to the same extent as pride; in fact, when compared with pride, anger was relatively more associated with low status. This distinction, between pride and anger expressions in conveying status, may relate to the specificity of the signal. Whereas the anger expression may represent a "quick and dirty" way of communicating aggression - a message that indirectly informs observers of the expresser's power and possible status - the pride expression may send a more distinct message of earned status, which could be based on aggressive power or on more affiliative behaviors such as leadership or task competence (Tracy et al., 2009). As a result, the overall message sent by anger is likely quite different from that sent by pride-observers may learn that the angry individual is powerful, but they will also be motivated to avoid him or her. Pride, in contrast, sends a clear message of high status and thus is unlikely to motivate avoidance; in fact, observers may choose to seek out proud individuals as valuable social group members. Future studies are needed to further address this issue by examining the responsive behaviors elicited by each expression, but the present research indicates that there is a distinction between the status associations of the two expressions.

Moreover, in contrast to Tiedens' (2001) finding that angry individuals are explicitly viewed as competent, the current results demonstrate that high-status perceptions of the pride expression are unelaborated and automatic. Indeed, this is the first research to suggest that our ability to rapidly and involuntarily assess social status may be due, in part, to our ability to automatically recognize and interpret displays of pride (Tracy \& Robins, 2008a). In the five IAT studies we conducted, participants faced conditions in which they viewed high-status words and expressions of pride, and were

\footnotetext{
${ }^{7}$ In one photo, the actor raised his arms up over his head, in a second he extended his arms out to the sides, and in the third he bent his arms upward at each elbow and raised them out to the sides.
} 
instructed to respond equally quickly to all stimuli. Yet, across studies, participants had markedly more difficulty inhibiting the association between pride and high status than inhibiting associations between pride and low status, and between other emotions (including anger and happiness) and high status. This suggests that understanding and interpreting the high-status message sent by the pride expression cannot be controlled. Thus, given that status is likely automatically perceived in many everyday social interactions, the present findings provide insight into how this process may work. Individuals may grant status to others implicitly, on the basis of their pride expressions, even in cases in which perceivers may wish to avoid doing so. The resultant knowledge and understanding gained, of the relative status of social group members, is likely an important part of interpersonal perception.

Finally, these studies suggest a novel application of the IAT and the AMP: The examination of functional signals sent by distinct emotion expressions. Given the strength of the effects found here, these methodologies hold promise for future research on emotion expressions. For example, researchers have long assumed that both anger and fear signal threat; implicit association studies could be used to determine whether this association is equally strong for both expressions. Studies could also examine how motivational state influences the way individuals perceive the messages sent by particular expressions; for example, does the association between the anger expression and threat depend on whether participants feel powerful versus weak?

\section{Limitations and Future Directions}

Researchers planning on using these implicit measures for studying emotion expression associations should, however, bear in mind several limitations. In Study 4, we followed Payne and colleagues (2005) in using the neutral gray box as the AMP's control stimulus. This decision introduces the possible alternate explanation that a picture of any person — regardless of expression —is more associated with high status than a neutral box. However, because we were already changing the attribute on which the painting were rated from simple liking or disliking to the more complex concept of status, we elected to match the other elements of the study to the original AMP as closely as possible. Nonetheless, future research should consider using a neutral but human image as a control.

Another caveat to be aware of is that, for all future studies using similar methodologies as well as for the present research, we cannot know whether the message sent by a particular expression is attributed to the sender, or whether any complex interpersonal judgment has been made. These measures only indicate that an implicit association exists between the expression and the message.

Several other issues limit the conclusions that can be drawn from this research, and should be addressed in future studies. First, all target photos featured the same individual: a 24 year-old White man. By including the same expresser across studies, we were able to make comparisons between conditions and studies. However, these findings should be replicated using female expressers and individuals of other ethnicities. Given that previous research has shown that status cues differ for males and females, and that pride recognition rates vary slightly depending on the gender and ethnicity of the individuals who show these expressions (Schmidt Mast \& Hall, 2004; Tracy \& Robins, 2008b), it is possible that the influence of these displays on perceived status may also be mod- erated by gender or ethnicity; furthermore, we might expect target by perceiver interactions. No perceiver-gender differences emerged in the present research, but this may have been due to the considerably fewer male participants included.

Second, all of the present studies relied on static and decontextualized images of emotion expressions. Although this format was chosen to maximize internal validity, future studies are needed to test whether the status associations of these expressions would be maintained under more externally valid conditions, in which individuals have access to a greater bandwidth of sensory cues as well as additional contextual information (such as whether the expresser has experienced a success or failure).

In conclusion, the present research suggests that the pride expression functions as a unique signal of high status, and is consistent with the suggestion that pride evolved to serve this purpose. However, alternative explanations for the present findings remain. It is possible, for example, that the pride-high-status association has become implicitly entrenched through cultural learning, although the recent finding that pride is spontaneously displayed following status-increasing events (i.e., achievement) even by the congenitally blind, who are unlikely to have learned the expression from cultural models, argues against this (Tracy \& Matsumoto, 2008). Nonetheless, evolutionary arguments for the pride-highstatus association will be considerably strengthened if the present findings generalize across cultures. Given the pan-human universality of status hierarchies (Buss, 2001) and the finding that the pride expression is reliably recognized in isolated nonliterate cultures (Tracy \& Robins, 2008b), the door is open for future studies to more directly address this issue.

\section{References}

Bargh, J. A. (1994). The four horsemen of automaticity: Awareness, intention, efficiency, and control in social cognition. In R. S. Wyer \& T. S. Srull (Eds.), Handbook of social cognition (Vol. 1, pp. 1-41). Hillsdale, NJ: Erlbaum.

Barrett, L. F. (2006). Emotions as natural kinds? Perspectives on Psychological Science, 1, 28-58.

Blanton, H., Jaccard, J., Klick, J., Mellers, B., Mitchell, G., \& Tetlock, P. (2009). Strong claims and weak evidence: Reassessing the predictive validity of the race IAT. Journal of Applied Psychology, 94, 567.

Buss, D. M. (2001). Human nature and culture: An evolutionary psychological perspective. Journal of Personality, 69, 955-978.

Cosmides, L., \& Tooby, J. (2000). Evolutionary psychology and the emotions. In M. Lewis \& J. M. Haviland-Jones (Eds.), Handbook of emotions (2nd ed., pp. 91-116). New York, NY: Guilford Press.

Darwin, C. (1872). The expression of emotion in man and animals. London, England: Murray.

de Waal, F. B. M. (1989). Chimpanzee politics: Power and sex among apes. Baltimore, MD: Johns Hopkins University Press.

Edelstein, R. S., \& Shaver, P. R. (2007). A cross-cultural examination of lexical studies of self-conscious emotions. In J. L. Tracy, R. W. Robins, \& J. P. Tangney (Eds.), The self-conscious emotions: Theory and research (pp. 194-208). New York, NY: Guilford Press.

Eibl-Eibesfeldt, I. (1989). Human ethology. New York: de Gruyter.

Ekman, P. (1992). An argument for basic emotions. Cognition and Emotion, 6, 169-200.

Ekman, P., \& Friesen, W. (1978). Facial action coding system: A technique for the measurement of facial movement: Investigator's guide 2 parts. Palo Alto: Consulting Psychologists.

Ekman, P., \& Friesen, W. V. (1971). Constants across cultures in the face and emotion. Journal of Personality and Social Psychology, 17, 124-129. 
Ekman, P., Friesen, W. V., O'Sullivan, M., Chan, A., DiacoyanniTarlatzis, I., Heider, K., et al. (1987). Universals and cultural differences in the judgments of facial expressions of emotion. Journal of Personality and Social Psychology, 53, 712-717.

Ekman, P., \& Rosenberg, E. L. (1998). What the face reveals. New York: Oxford University Press.

Fessler, D. M. T. (1999). Toward an understanding of the universality of second-order emotions. In A. L. Hinton (Ed.), Biocultural approaches to the emotions: Publications of the Society for Psychological Anthropology (pp. 75-116). New York: Cambridge University Press.

Greenwald, A. G., \& Banaji, M. R. (1995). Implicit social cognition: Attitudes, self-esteem, and stereotypes. Journal of Personality and Social Psychology, 102, 4-27.

Greenwald, A. G., \& Farnham, S. D. (2000). Using the Implicit Association Test to measure self-esteem and self-concept. Journal of Personality and Social Psychology, 79, 1022-1038.

Greenwald, A. G., McGhee, D. E., \& Schwartz, J. L. K. (1998). Measuring individual differences in implicit cognition: The Implicit Association Test. Journal of Personality and Social Psychology, 74, 1464-1480.

Greenwald, A. G., Nosek, B. A., \& Banaji, M. R. (2003). Understanding and using the Implicit Association Test 1: An improved scoring algorithm. Journal of Personality and Social Psychology, 85, 197-216.

Haidt, J., \& Keltner, D. (1999). Culture and emotion: Multiple methods find new faces and a gradient of recognition. Cognition and Emotion, 13, 225-266.

Heerey, E. A., Keltner, D., \& Capps, L. M. (2001). Making sense of self-conscious emotion: Linking theory of mind in children with autism. Emotion, 3, 394-400.

Keltner, D. (1995). Signs of appeasement: Evidence for the distinct displays of embarrassment, amusement, and shame. Journal of Personality and Social Psychology, 68, 441-454.

Keltner, D., \& Ekman, P. (2000). Facial expression of emotion. In M. Lewis \& J. M. Haviland-Jones (Eds.), Handbook of emotions (2nd ed., pp. 236-249). New York: Guilford Press.

Keltner, D., Gruenfeld, D. H., \& Anderson, C. (2003). Power, approach, and inhibition. Psychological Review, 110, 265-284.

Keltner, D., Young, R. C., \& Buswell, B. N. (1997). Appeasement in human emotion, social practice, and personality. Aggressive Behavior, $23,359-374$

Kipfer, B. A. (2005). Roget's 21st century thesaurus (3rd ed.). New York: Dell.

Knutson, B. (1996). Facial expressions of emotion influence interpersonal trait inferences. Journal of Nonverbal Behavior, 20, 165-182.

Lewis, M., Alessandri, S. M., \& Sullivan, M. W. (1992). Differences in shame and pride as a function of children's gender and task difficulty. Child Development, 63, 630-638.

Maison, D., Greenwald, A. G., \& Bruin, R. H. (2004). Predictive validity of the implicit association test in studies of brands, consumer attitudes, and behavior. Journal of Consumer Behaviour, 14, 405-418.

Mogg, K., \& Bradley, B. P. (1999). Some methodological issues in assessing attentional biases for threatening faces in anxiety: A replication study using a modified version of the probe detection task. Behaviour Research and Therapy, 37, 595-604.

Ohman, A. (2002). Automaticity and the amygdala: Nonconscious responses to emotional faces. Current Directions in Psychological Science, 11, 62-66.

Ohman, A., \& Mineka, S. (2001). Fears, phobias, and preparedness: Toward an evolved module of fear and fear learning. Psychological Review, 108, 483-522.

Payne, B. K. (2005). Conceptualizing control in social cognition: How executive functioning modulates the expression of automatic stereotyping, Journal of Personality and Social Psychology, 89, 488-503.

Payne, B. K., Cheng, C. M., Govorun, O., \& Stewart, B. D. (2005). An inkblot for attitudes: Affect misattribution as implicit measurement. Journal of Personality and Social Psychology, 89, 277.

Penke, L., Eichstaedt, J., \& Asendorpf, J. B. (2006). Single Attribute Implicit Association Tests (SA-IAT) for the assessment of unipolar constructs: The case of sociosexuality. Experimental Psychology, 53, 283-291.

Russell, J. A. (2003). Core affect and the psychological construction of emotion. Psychological Review, 110, 145-172.

Schmid Mast, M., \& Hall, J. A. (2004). Who is the boss and who is not? Accuracy of judging status. Journal of Nonverbal Behavior, 28, 145-165.

Sedikides, C., \& Skowronski, J. J. (1997). The symbolic self in evolutionary context. Personality and Social Psychology Review, 1, 80-102.

Shiota, M. N., Campos, B., Keltner, D., \& Hertenstein, M. J. (2004). Positive emotion and the regulation of interpersonal relationships. In P. Philippot \& R. S. Feldman (Eds.), The regulation of emotion (pp. 125-155). Mahwah, NJ: Erlbaum.

Tiedens, L. Z. (2001). Anger and advancement versus sadness and subjugation: The effect of negative emotion expressions on social status conferral. Journal of Personality and Social Psychology, 80, 86-94.

Tiedens, L., Ellsworth, P. C., \& Mesquita, B. (2000). Sentimental stereotypes: Emotional expectations for high- and low-status group members. Personality and Social Psychology Bulletin, 26, 560-575.

Tiedens, L. Z., Ellsworth, P. C., \& Moskowitz, D. S. (2000). Feeling high and feeling low: Emotional consequences of social status position. Manuscript submitted for publication.

Tierney, J. (2008). In bias test, shades of gray. The New York Times Retrieved September 10, 2009, from http://www.nytimes.com/2008/11/ 18/science/18tier.html

Tracy, J. L., \& Matsumoto, D. (2008). The spontaneous display of pride and shame: Evidence for biologically innate nonverbal displays. Proceedings of the National Academy of Sciences USA, 105, 11655-11660.

Tracy, J. L., \& Robins, R. W. (2004). Show your pride: Evidence for a discrete emotion expression. Psychological Science, 15, 194-197.

Tracy, J. L., \& Robins, R. W. (2007a). Emerging insights into the nature and function of pride. Current Directions in Psychological Science, 16, 147-150.

Tracy, J. L., \& Robins, R. W. (2007b). The prototypical pride expression: Development of a nonverbal behavioral coding system. Emotion, 7, 789-801.

Tracy, J. L., \& Robins, R. W. (2008a). The automaticity of emotion recognition. Emotion, 8, 81-95.

Tracy, J. L., \& Robins, R. W. (2008b). The nonverbal expression of pride: Evidence for cross-cultural recognition. Journal of Personality and Social Psychology, 94, 516-530.

Tracy, J. L., Robins, R. W., \& Schriber, R. A. (2009). Development of a FACS-verified set of basic and self-conscious emotions expressions. Emotion, 9, 554-559.

Tracy, J. L., Shariff, A. F., \& Cheng, J. T. (in press). A naturalist's view of pride. Emotion.

Weisfeld, G. E., \& Beresford, J. M. (1982). Erectness of posture as an indicator of dominance or success in humans. Motivation and Emotion, $6,113-131$.

Williams, L. A., \& DeSteno, D. (2008). Pride and perseverance: The motivational role of pride. Journal of Personality and Social Psychology, 94, 1007-1017.

Williams, L. A., \& DeSteno, D. (2009). Pride: Adaptive social emotion or seventh sin? Psychological Science, 20, 284-288.

Winkielman, P., \& Berridge, K. C. (2003). Unconscious affective reactions to masked happy versus angry faces influence consumption behavior and judgments of value. Personality and Social Psychology Bulletin, 31, $121-135$.

Received February 12, 2009

Revision received May 14, 2009

Accepted May 15, 2009 